\title{
EQUIVALENCIA TERAPÉUTICA EVALUADA MEDIANTE ESTUDIOS IN VITRO DE MEDICAMENTOS MULTIFUENTES: ESTUDIO DE CASOS DE AMOXICILINA, DOXICICLINA Y FLUCONAZOL EN LIMA, PERÚ
}

Miguel Grande-Ortiz",a , Sandra Taipe-Cadenillas²,b, Cinthia Villodas-Saldaña ${ }^{2, b}$, Javier Rodríguez-Calzado,c . Luis Moreno-Exebio ${ }^{1, d}$

\begin{abstract}
RESUMEN
El objetivo del estudio fue determinar la equivalencia terapéutica evaluada mediante estudios in vitro de cuatro marcas de medicamentos conteniendo amoxicilina, doxiciclina y fluconazol adquiridos en establecimientos farmacéuticos de Lima Metropolitana y establecer su intercambiabilidad con un producto de referencia (PR). Se empleó un método validado de espectrofotometría ultravioleta visible para determinar el perfil de disolución. El factor de similitud (f2) se utilizó para establecer la equivalencia terapéutica, considerándose equivalentes si los valores de f2 se encontraban entre 50 y 100. Para doxiciclina los cuatro medicamentos fueron equivalentes in vitro al PR, para amoxicilina sólo dos medicamentos fueron equivalentes in vitro al PR y para fluconazol ninguno fue equivalente in vitro al PR. Se concluye que algunos medicamentos de amoxicilina y fluconazol que circulan en el mercado nacional no cumplen con la equivalencia terapéutica evaluada mediante estudios in vitro; es decir, no son intercambiables.
\end{abstract}

Palabras claves: Equivalencia Terapéutica; Medicamentos genéricos; Biofarmacéutica; Medicamentos bioequivalentes; Disolución (fuente: DeCS BIREME)

\section{THERAPEUTIC EQUIVALENCE EVALUATED BY IN VITRO STUDIES OF MULTISOURCE PHARMACEUTICAL PRODUCTS: CASE STUDIES OF AMOXICILLIN, DOXYCYCLINE AND FLUCONAZOLE IN LIMA, PERU}

\begin{abstract}
The objective of the study was to determine the therapeutic equivalence evaluated through in vitro studies of four brands of drugs containing amoxicillin, doxycycline, and fluconazole purchased at pharmaceutical facilities in Metropolitan Lima, and to establish their interchangeability with a reference product (RP). A validated method of visible ultraviolet spectrophotometry was used to determine the dissolution profile. The similarity factor (f2) was used to establish the therapeutic equivalence, being considered equivalent if the values of $\mathrm{f} 2$ were between 50 and 100 . For doxycycline, the four drugs were equivalent in vitro to the RP; for amoxicillin, only two drugs were equivalent in vitro to the RP; and for fluconazole, none was equivalent in vitro to the RP. It is concluded that some amoxicillin and fluconazole drugs circulating in the national market do not meet the therapeutic equivalence assessed by in vitro studies; in other words, they are not interchangeable.
\end{abstract}

Keywords: Therapeutic Equivalence; Generic drugs; Biopharmaceutical; Bioequivalent drugs; Dissolution (source: MeSH NLM).

\section{INTRODUCCIÓN}

La Organización Mundial de la Salud (OMS) establece que las personas deben tener acceso a medicamentos esenciales seguros, efectivos y de calidad; y su prescripción obedece a un uso racional ${ }^{(1)}$.
A través de la adopción de políticas farmacéuticas y estrategias a nivel nacional, los gobiernos establecen el marco que asegura el acceso equitativo y sostenible de medicinas y tecnologías de la salud. El centro de estas políticas y estrategias es el principio de calidad, seguridad y eficacia ${ }^{(2)}$. Los medicamentos multifuentes (MM) deben

\footnotetext{
Centro Nacional de Control de Calidad, Instituto Nacional de Salud. Lima, Perú.

Universidad Privada Norbert Wiener. Lima, Perú.

Químico farmacéutico; ${ }^{b}$ bachiller en Farmacia y Bioquímica; ${ }^{\mathrm{c}}$ biólogo; ${ }^{\mathrm{d}}$ doctor en Farmacia y Bioquímica

Recibido: 05/09/2018 Aprobado: 27/02/2019 En línea: 21/03/2019
}

Citar como: Grande-Ortiz M, Taipe-Cadenillas S, Villodas-Saldaña C, Rodríguez-Calzado J, Moreno-Exebio L. Equivalencia terapéutica evaluada mediante estudios in vitro de medicamentos multifuentes: Estudio de casos de amoxicilina, doxiciclina y fluconazol en Lima, Perú. Rev Peru Med Exp Salud Publica. 2019;36(1):74-80. doi:10.17843/rpmesp.2019.361.3912. 
tener la característica de ser intercambiables; es decir, terapéuticamente equivalentes al producto innovador ${ }^{(3)}$.

Una opción para establecer la equivalencia terapéutica es mediante los perfiles de disolución, donde se puede predecir la liberación del fármaco in vivo. Esto se aplica para ciertos tipos de fármacos altamente solubles de acuerdo con el Sistema de Clasificación Biofarmacéutica (BCS) ${ }^{(4,5)}$.

El objetivo del presente estudio fue determinar la equivalencia terapéutica mediante estudios in vitro de cuatro marcas diferentes de medicamentos conteniendo amoxicilina, doxiciclina y fluconazol, adquiridos en establecimientos farmacéuticos de Lima Metropolitana, a los cuales se les comparó con un producto de referencia para establecer su intercambiabilidad ${ }^{(6-8)}$.

\section{EL ESTUDIO}

El estudio fue analítico, experimental y transversal. La muestra estuvo constituida por 200 cápsulas del producto de referencia y de los MM conteniendo amoxicilina, doxiciclina y fluconazol, adquiridos en establecimientos farmacéuticos de Lima Metropolitana, todas ellas del mismo lote, de las cuales 100 fueron usadas para los ensayos de calidad y de perfil de disolución. Las restantes quedaron como contramuestra.

\section{REACTIVOS}

Se utilizaron estándares primarios de amoxicilina, doxiciclina y fluconazol de la USP (United States Pharmacopeia); acetonitrilo (J.T. Baker, USA) y metanol grado HPLC (J.T. Baker, México), fosfato de potasio monobásico, ácido fosfórico, edetato disódico, trietilamina, ácido clorhídrico, cloruro de sodio, acetato de sodio anhidro, ácido acético, hidróxido de sodio (Merck KGaA, Darmstadt, Germany) fueron de grado analítico y agua grado $\operatorname{HPLC}(18,2 \mathrm{M} \Omega)$ obtenida a través de un equipo purificador de agua Milli $Q$ (Millipore Advantage A10). Todos los reactivos fueron de calidad ACS (American Chemical Society).

\section{ENSAYOS DE CONTROL DE CALIDAD}

Los medicamentos fueron sometidos a los siguientes ensayos de control de calidad: peso promedio; identificación y contenido, disolución y uniformidad de contenido; previo al perfil de disolución.

\section{Peso Promedio (variación de peso)}

El peso de cápsulas fue registrado para cada producto analizado. La variación de peso fue calculada como la desviación estándar (s), usando la ecuación:

$$
s=\sqrt{\frac{\sum_{i}^{n}=1\left(x_{i}-\bar{x}\right)^{2}}{n-1}}
$$

\section{MENSAJES CLAVE}

Motivación para realizar el estudio. La falta de información respecto a la calidad y eficacia de los medicamentos multifuentes que se comercializan en el Perú, constituye un problema de salud pública.

Principales hallazgos. El presente estudio encontró que todos los medicamentos multifuentes de doxiciclina fueron equivalentes in vitro al producto de referencia, solo dos de cuatro medicamentos multifuentes conteniendo amoxicilina fueron equivalentes in vitro al producto de referencia y ninguno de los medicamentos multifuentes evaluados conteniendo fluconazol fue equivalente in vitro al innovador.

Implicancias. Estos resultados muestran la necesidad de aplicar la normatividad vigente en este tema por parte de la autoridad regulatoria.

Donde $x_{i}$ son los pesos individuales, $\bar{x}$ es la media de todos los pesos y $n$ es el número de muestras utilizadas. La variación de peso fue registrada para evaluar si algún valor muestra pesos altos o bajos asociados a sobredosificación o subdosificación de las unidades analizadas. El peso promedio fue realizado según la Farmacopea Británica (BP) 2013 Apéndice XII C ${ }^{(9)}$.

Identificación y contenido, disolución y uniformidad de contenido

Los ensayos de identificación y contenido, disolución y uniformidad por variación de peso de amoxicilina y doxiciclina fueron realizados según la United States Pharmacopeia (USP) 37, capítulos generales <621>, <711> y <905>, respectivamente. El ensayo de identificación y contenido, disolución y uniformidad por variación de peso de fluconazol, fueron realizados según técnica propia del laboratorio fabricante ${ }^{(10)}$.

\section{PERFIL DE DISOLUCIÓN}

\section{Preparación de los medios de disolución}

El fluido gástrico simulado $\mathrm{pH} 1,2$, buffer acetato $\mathrm{pH} 4,5$ y fluido intestinal simulado $\mathrm{pH} \mathrm{6,8}$; fueron preparados de acuerdo a las instrucciones de la Farmacopea Internacional, cuarta edición (11). Todos los medios fueron preparados sin enzimas. Los medios fueron filtrados con nylon 0,45 $\mu \mathrm{m}$ y desgasificados al vacío con agitación mecánica.

\section{Equipos}

Se utilizó un equipo de Disolución Vankel (VK 7025 Varian) de ocho vasos con automuestrador y enlazado en línea con un espectrofotómetro UV-VIS (Cary 50). El aparato de disolución 2 USP (Paletas) a 75 rpm y $900 \mathrm{~mL}$ de medio de disolución fueron empleados en todos los ensayos. Se utilizaron sinkers en todos los casos. Las cantidades disueltas fueron determinadas por espectrofotometría UV- 
VIS, tomando como referencia el Reporte Técnico 937, de la Organización Mundial de la Salud (OMS) ${ }^{(5)}$. La toma de muestra fue automatizada sin reposición. En el caso de amoxicilina los puntos de muestreo fueron: 5,10, 15, 30, 45, 60 y 75 minutos; para doxiciclina y fluconazol los puntos de muestreo fueron $5,10,15,20,30$ y 45 minutos.

\section{Medicamentos multifuentes evaluados}

La Tabla 1 muestra todos los productos conteniendo amoxicilina, doxiciclina y fluconazol. Todos los productos fueron evaluados al menos 12 meses antes de su fecha de expiración.

\section{CUANTIFICACIÓN ANALÍTICA}

\section{Amoxicilina}

La cuantificación analítica de las muestras de disolución fue realizada por espectrofotometría (UV-Vis) a $272 \mathrm{~nm}$, usando una celda de $0,5 \mathrm{~cm}$ de longitud, las condiciones se realizaron de acuerdo a la monografía de la USP 37 para amoxicilina cápsulas. El coeficiente de correlación de la curva de calibración fue al menos 0,999 para cada medio y los coeficientes de variación de los factores de respuesta fueron 1,778 (pH 1,2), 1,623 (pH 4,5) y 1,972 (pH 6,8).

\section{Doxiciclina}

La cuantificación analítica de las muestras de disolución fue realizada por espectrofotometría (UV-Vis) a $276 \mathrm{~nm}$, usando una celda de $1,0 \mathrm{~cm}$ de longitud, las condiciones se realizaron de acuerdo a la monografía de la USP 37 para doxiciclina hiclato cápsulas. El coeficiente de correlación de la curva de calibración fue al menos 0,999 para cada medio y los coeficientes de variación de los factores de respuesta fueron 1,515 (pH 1,2), 0,927 (pH 4,5) y 0,592 (pH 6,8).

\section{Fluconazol}

La cuantificación analítica de las muestras de disolución fue realizada por espectrofotometría (UV-Vis) a $261 \mathrm{~nm}$, usando una celda de $1,0 \mathrm{~cm}$ de longitud, las condiciones se realizaron de acuerdo a la metodología del PR. El coeficiente de correlación de la curva de calibración fue al menos 0,999 para cada medio y los coeficientes de variación de los factores de respuesta fueron $1,335(\mathrm{pH}$ $1,2), 0,985(\mathrm{pH} \mathrm{4,5})$ y 1,351 (pH 6,8).

\section{VALIDACIÓN DE LOS MÉTODOS DEL ESTUDIO}

La validación de los métodos para el desarrollo de los perfiles de disolución se realizó con los siguientes parámetros: linealidad, precisión, exactitud, especificidad, estabilidad e influencia del filtro, de acuerdo a la Guía Técnica G-BIOF 02 Bioexención de los estudios de Biodisponibilidad / Bioequivalencia para establecer Equivalencia Terapéutica de Formas Farmacéuticas Sólidas del Instituto de Salud Pública de Chile ${ }^{(12)}$ (Anexo 1 y 2 - visualizar en versión electrónica).

\section{SELECCIÓN DEL PRODUCTO DE REFERENCIA}

El producto de referencia fue escogido de acuerdo a los criterios de la OMS ${ }^{(5)}$, el PR fue Amoxil 500 mg cápsulas (Glaxo SmithKline). Para doxiciclina fue Vibramicina $100 \mathrm{mg}$ cápsulas (Pfizer Inc.) y para fluconazol fue Diflucan $150 \mathrm{mg}$ cápsulas (Pfizer Inc.).

\section{ANÁLISIS ESTADÍSTICO}

Se evaluaron doce tabletas de cada formulación por lote y todos los datos de disolución fueron evaluados usando las hojas de cálculo validadas del Centro Nacional de Control de Calidad del Instituto Nacional de Salud. Cada MM fue comparado con el PR de acuerdo con el siguiente criterio: si la disolución es muy rápida, es decir más del $85 \%$ del

Tabla 1. Medicamentos evaluados que contienen amoxicilina, doxiciclina y fluconazol

\begin{tabular}{lccc}
\hline Producto & País & Producto & Lote \\
\hline & Reino Unido & Referencia & 020 CQ079V \\
Amoxicilina & Perú & Multifuente A & 10401584 \\
& Italia & Multifuente B & 4 D03114 \\
& Colombia & Multifuente C & 431013 \\
& Perú & Multifuente D & 1110454 \\
México & Referencia & 1458006 \\
& Perú & Multifuente A & 1090414 \\
& Perú & Multifuente B & 10467744 \\
& Perú & Multifuente C & 1042114 \\
Fluconazol & Perú & Multifuente D & 110024 \\
& Brasil & Referencia & $40492004 \mathrm{~A}$ \\
& Perú & Multifuente A & 1110324 \\
& Perú & Multifuente B & 10717634 \\
& Perú & Multifuente C & 11002294 \\
\hline
\end{tabular}

A, B, C y D corresponden a cuatro fabricantes diferentes de medicamentos multifuentes 
Tabla 2. Cálculo del factor de similitud $\left(\mathrm{f}_{2}\right)$ de las diferentes formulaciones de amoxicilina

\begin{tabular}{lcccc}
\hline & Multifuente A & Multifuente B & Multifuente C & Multifuente D \\
\hline $\mathrm{pH} 1,2$ & \multicolumn{4}{c}{ No aplica* $^{*}$} \\
$\mathrm{pH} 4,5$ & 48,9 & 50,8 & 52,4 & 45,4 \\
$\mathrm{pH} \mathrm{6,8}$ & 54,7 & 50,5 & 52,3 & 66,7 \\
Resultados & No Equivalente & Equivalente & Equivalente & No Equivalente \\
\hline
\end{tabular}

A, B, C y D corresponden a cuatro fabricantes diferentes de medicamentos multifuentes

${ }^{*} \mathrm{f}_{2}$ no fue calculado porque la disolución fue muy rápida

$(50 \leq \mathrm{f} 2 \leq 100)$

medicamento se disuelve en menos de 15 minutos, no se efectúa cálculo de $\mathrm{f}_{2}$ y se asume que el genérico es equivalente al innovador ${ }^{(5)}$.

La comparación de los perfiles de disolución fue realizada de acuerdo al Reporte Técnico 937, de la Organización Mundial de la Salud (OMS) ${ }^{(5)}$, calculando el factor de similitud $\left(f_{2}\right)$, según ecuación que se muestra a continuación:

Donde $R_{t}$ y $T_{t}$ son los porcentajes promedios disueltos en

$$
f_{2}=50 * \log \left\{\left|1+\frac{1}{n} \sum_{t=1}^{n}\left(R_{t}-T_{t}\right)^{2}\right|^{-0,5} * 100\right\}
$$

cada tiempo del medicamento de referencia y en estudio y $n$ es el número de tiempos de muestreo. Para establecer la equivalencia terapéutica se consideró que dos formulaciones presentan equivalencia terapéutica evaluada mediante estudios in vitro si los valores $f_{2}$ estaban comprendidos entre 50 y 100. Para el análisis estadístico se utilizó el programa Microsoft Excel versión 2014.

\section{HALLAZGOS}

\section{AMOXICILINA}

El PR pasó los requerimientos de calidad y el ensayo de uniformidad <905> de la USP. La variación de peso de las cápsulas fue entre 604,6 y $631,0 \mathrm{mg}$. Las desviaciones estándar para los productos variaron entre 1,13 y 10,66 . El contenido de amoxicilina PR fue $98,7 \%$ y de los medicamentos multifuentes fue 102,3\% (A), 97,6\% (B), $99,6 \%$ (C), 99,3\% (D). El rango de aceptación es 90,0\% $120,0 \%$

La Figura 1, muestra el comportamiento de los perfiles de disolución de los productos que contienen amoxicilina vendidos en Perú versus los datos del PR. El PR, el genérico $\mathrm{B}$ y el genérico $\mathrm{C}$ se disolvieron rápidamente en los tres medios y fueron considerados equivalentes in vitro. Los genéricos $A$ y $D$ disolvieron en menos de $85 \%$ en 15 minutos en $\mathrm{pH} 4,5$ y no pasaron la prueba $\mathrm{f}_{2}$ de comparación con el PR. La tabla 2 resume la comparación $\left(f_{2}\right)$ entre los diferentes productos de amoxicilina y el PR.

\section{DOXICICLINA}

EI PR pasó los requerimientos de calidad y el ensayo de uniformidad <905> de la USP. La variación de peso de las cápsulas fue entre 244,0 y $280,7 \mathrm{mg}$. Las desviaciones estándar para los productos variaron entre 2,28 y 8,90. El contenido de doxiciclina PR fue $104,9 \%$ y de los medicamentos multifuentes fue 100,8\% (A), 105,7\% (B), $106,7 \%$ (C), 108,9\% (D). El rango de aceptación es 90,0\% $120,0 \%$.

La Figura 1 muestra el comportamiento de los perfiles de disolución de los productos que contienen doxiciclina vendidos en el Perú versus los datos del PR. El PR y todos los productos se disolvieron rápidamente en los tres medios y se consideraron equivalentes in vitro, de acuerdo con la guía de la OMS ${ }^{(5)}$.

El factor de similitud $\left(f_{2}\right)$ entre los diferentes productos de doxiciclina y el PR no fue calculado porque la disolución fue muy rápida en los tres $\mathrm{pH}$; por tanto, las cuatro formulaciones son equivalentes al PR.

\section{FLUCONAZOL}

EI PR pasó los requerimientos de calidad y el ensayo de uniformidad <905> de la USP. La variación de peso de las cápsulas fue entre 303,3 y 389,3 mg. Las desviaciones estándar para los productos variaron entre 0,77 y 12,93 . El contenido de Fluconazol PR fue $101,8 \%$ y de los medicamentos multifuentes fue $105,0 \%$ (A), 102,4\% (B), 99,8\% (C), 100,3\% (D). El rango de aceptación es $95,0 \%-105,0 \%$.

La Figura 1 muestra el comportamiento de los perfiles de disolución de los productos conteniendo fluconazol vendidos en el Perú versus los datos del PR. Ninguno de los productos analizados mostró equivalencia in vitro al $\mathrm{PR}$.

La Tabla 3 resume la comparación $\left(\mathrm{f}_{2}\right)$ entre los diferentes productos de fluconazol y el PR.

\section{DISCUSIÓN}

Según los resultados de control de calidad, los medicamentos que contienen amoxicilina y doxiciclina, cumplen con los criterios de calidad según la USP 37, igualmente los medicamentos que contienen fluconazol cumplen con las especificaciones del laboratorio de origen. En todos los casos se cumplió con el requisito de la OMS ${ }^{(5)}$ para la realización de 
Tabla 3. Cálculo del factor de similitud $\left(\mathrm{f}_{2}\right)$ de las diferentes formulaciones de fluconazol

\begin{tabular}{lcccc}
\hline & Multifuente A & Multifuente B & Multifuente C & Multifuente D \\
\hline $\mathrm{pH} 1,2$ & 29,2 & 17,3 & 23,3 & 25,4 \\
$\mathrm{pH} 4,5$ & 17,9 & 8,2 & 11,6 & 9,7 \\
$\mathrm{pH} 6,8$ & 18,2 & 8,9 & 11,4 & 9,1 \\
Resultados & No Equivalente & No Equivalente & No Equivalente & No Equivalente \\
\hline
\end{tabular}

A, B, C y D corresponden a cuatro fabricantes diferentes de medicamentos multifuentes $(50 \leq \mathrm{f} 2 \leq 100)$

los perfiles de disolución: «La diferencia entre el porcentaje del contenido del principio activo del producto de referencia y de los MM no fue mayor a $5 \%$.

En relación con la amoxicilina, todos los MM presentaron una disolución rápida a pH 1,2, lo que confirma su clasificación en el grupo I (BCS) y sería considerado elegible a un estudio de bioexención ${ }^{(5)}$. En el pH 4,5, los MM A y D evidenciaron una lenta disolución en comparación con los otros medicamentos evaluados. Esta variación puede deberse a los diferentes métodos de manufactura, formulación y empaque utilizado ${ }^{(8)}$.
En un estudio multicéntrico (Perú, Chile y Argentina) realizado

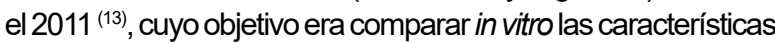
de disolución de medicamentos que contienen amoxicilina, metronidazol y zidovudina; se encontró que sólo uno de tres marcas de amoxicilina tabletas $500 \mathrm{mg}$ que se comercializaban en el Perú en ese momento, demostró ser equivalente con el producto de referencia.

Respecto a doxiciclina, todos los medicamentos presentaron una disolución muy rápida a $\mathrm{pH} 1,2 ; 4,5$ y 6,8. Una disolución de $85 \%$ en 15 minutos a los tres $\mathrm{pH}$ señalados puede asegurar que la biodisponibilidad del fármaco no esté
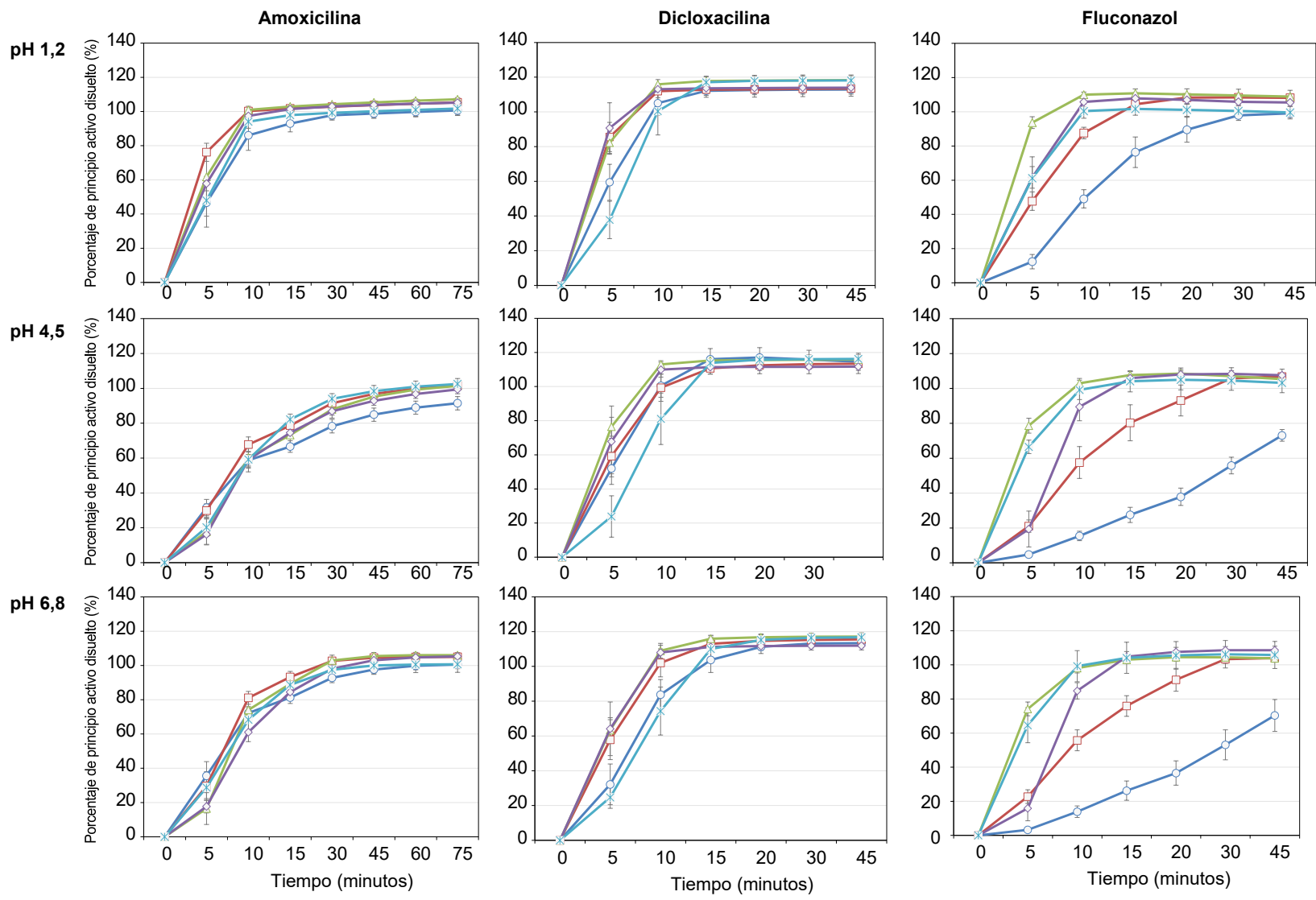

$\succ$ Multifuente $D \sim$ Multifuente C

$\triangle$ Multifuente B

$\rightarrow-$ Multifuente A $\quad-$ - Referencia

Figura 1. Comportamiento de la disolución del producto de referencia y medicamentos conteniendo amoxicilina, doxiciclina y fluconazol comercializados en el Perú, en los tres niveles de pH evaluados. A, B, C y D corresponden a cuatro fabricantes diferentes de medicamentos multifuentes. 
limitada por la disolución. En estos casos, el paso limitante de velocidad de la absorción del fármaco es el vaciamiento gástrico ${ }^{(5)}$.

Estos resultados se correlacionaron con un estudio para comparar los perfiles de disolución de cinco marcas de doxiciclina con el innovador, realizado en Pakistán en el 2014, encontrándose que todos los genéricos se disuelven en más del $85 \%$ en 15 minutos en los tres $\mathrm{pH}$, con lo cual se demuestra que estos son terapéuticamente equivalentes con el innovador ${ }^{(14)}$.

Sobre el fluconazol, ninguno de los cuatro MM evaluados desarrolló perfiles de disolución similares al producto de referencia, el cual fue fabricado en Brasil, país donde es requisito realizar estudios de equivalencia terapéutica para el registro sanitario de los medicamentos.

En un estudio realizado en Ecuador en $2013^{(16)}$, se encontró que sólo dos de cuatro medicamentos conteniendo fluconazol fueron equivalentes al producto innovador, este estudio utilizó el mismo innovador que el nuestro.

El fluconazol es un agente de primera línea para el tratamiento y profilaxis de infecciones por Candida albicans, sobre todo en pacientes con VIH y en pacientes con cáncer que reciben agentes citostáticos, por ello es importante garantizar la eficacia de este medicamento ${ }^{(17)}$.

Este estudio demuestra que existen productos en el mercado peruano que no presentan intercambiabilidad con el PR. Sólo analizamos cuatro MM de amoxicilina y fluconazol, pero existen registrados $186 \mathrm{MM}$ de amoxicilina y 162 MM de fluconazol en la Dirección General de Medicamentos, Insumos y Drogas ${ }^{(18)}$. Esto significa que existen muchos productos de amoxicilina y fluconazol cuya seguridad y eficacia es desconocida.
Es importante señalar que el Ministerio de Salud, aprobó recientemente el reglamento que regula la intercambiabilidad de medicamentos en el Perú (D.S. N. ${ }^{\circ}$ 024-2018-SA), en el se señala las condiciones y exigencias para realizar los estudios de equivalencia terapéutica in vitro e in vivo, constituyendo una exigencia legal para el registro sanitario de los medicamentos consignados en dicha normativa ${ }^{(19)}$.

Una limitación fue la falta de disponibilidad de los productos de referencia, se pudo obtener amoxicilina de un país de alta vigilancia sanitaria, pero no doxiciclina ni fluconazol; sin embargo, Brasil y México son países donde el requisito de bioequivalencia forma parte de su legislación ${ }^{(20)}$. Otra limitación fue la inclusión de un solo lote de cada producto estudiado, por un tema presupuestal y porque en ese tiempo no estaba aprobada la normativa nacional, que considera actualmente dos lotes.

En conclusión, todos los MM de doxiciclina mostraron equivalencia in vitro al PR. Sólo dos de cuatro genéricos de amoxicilina presentaron equivalencia in vitro al PR. Ninguno de los genéricos de fluconazol exhibió equivalencia in vitro al PR.

Contribuciones de autoría: MGO participó en el análisis e interpretación de datos, revisión y aprobación de la versión final del artículo, LEME participó en la concepción y diseño del artículo, redacción, revisión y aprobación de la versión final del artículo. STC y CVS, han participado en la recolección de datos, análisis e interpretación de datos y revisión crítica del artículo. JRC participó en el análisis estadístico e interpretación de datos y revisión crítica del artículo.

Financiamiento: El presente trabajo fue realizado con fondos provenientes del Instituto Nacional de Salud de Perú.

Conflictos de Interés: Los autores declaran no tener conflicto de interés en la publicación de este artículo

\section{REFERENCIAS BIBLIOGRÁFICAS}

1. World Health Organization. Annual Report WHO Essential Medicines \& Health Products 2016 [Internet]. Geneva: WHO;2016.Disponibleen: http://www. who.int/medicines/publications/annualreports/WHO_EMP_Report_2016_ Online.pdf ?ua $=\overline{1}$

2. Bolaños R, Bond K, Child R, Coto JV, Cummings R, Dellepiane N, et al. Improving safety, quality and efficacy in the Americas. Rev Panam Salud Publica. 2016;39(5):215-6.

3. Laosa O, Guerra P, López-Durán J, Mosquera B, Frías J. Estudios de Bioequivalencia: La necesidad de establecer la fiabilidad de los medicamentos multifuentes. Rev Perú Med Exp Salud Pública 2009; 26(4):553-62.
4. Amidon G, Lennernas H, Shah VP, Crison JR. A Theoretical basis for a Biopharmaceutic Drug Classification. The Correlation of in vitro Drug Product Dissolution and in vivo Bioavailability. AAPS Journal 2014; 16(5):894-898. doi: $10.1208 / \mathrm{s} 12248-014-9620-9$.

5. World Health Organization. Fortieth report. Annex 8: Proposal to waive in vivo bioequivalence requirements for WHO model list of essential medicinesinmediate-release, solid oral dosage forms. Geneva: WHO; 2006. Disponible en: http://apps.who.int/medicinedocs/ documents/s14091e/s14091e.pdf

6. U.S. Department of Health and Human Services, Food and Drug Administration, Center for Drug Evaluation and Research
(CDER). Waiver of In Vivo Bioavailability and Bioequivalence Studies for Immediate-Release Solid Oral Dosage Forms Based on a Biopharmaceutics Classification System Guidance for Industry [Internet]. New Hampshire: HHS, FDA, CDER; 2017. Disponible en: https:// www.fda.gov/downloads/Drugs/GuidanceComplianceRegulatoryInformation/ Guidances/UCM070246.pdf

7. European Medicines Agency. Guideline on the investigation of bioequivalence [Internet]. London: EMEA; 2010. Disponible en: http://www.ema. europa.eu/docs/en_GB/document_library/Scientific_guideline/2010/01/ WC500070039.pdf

8. Ministerio de Salud. Petitorio Nacional Único de Medicamentos Esenciales para 
el Sector Salud. Lima: MINSA; 2015. Disponible en: http://www.digemid. minsa.gob.pe/UpLoad/UpLoaded/PDF/ Normatividad/2015/RM_399-2015.pdf

9. British Pharmacopoeia Commission. British Pharmacopeia. London: The Stationery Office; 2013.

10. The United States Pharmacopeial Convention. US Pharmacopoeia, 37ed. 2014.

11. World Health Organization. Department of Essential Medicines and Pharmaceutical Policies (EMP). The International Pharmacopoeia Fourth Edition; 2011.

12. Instituto de Salud Pública de Chile. Guía Técnica G-Biof 02: Bioexención de los estudios de Biodisponibilidad y Bioequivalencia para establecer Equivalencia Terapéutica de formas farmacéuticas sólidas orales [Internet]. Santiago: ISP; 2007. Disponible en http://www.ispch.cl/sites/ default/files/guia_tec_g_biof02.pdf

13. Löbenberg R, Chacra NB, Stippler ES, Shah VP, DeStefano AJ, Hauck WW, et al. Toward Global Standards for Comparator Pharmaceutical Products: Case Studies of Amoxicillin, Metronidazole and Zidovudine in the Americas. The AAPS Journal 2012. 14 (3):462-71. doi: 10.1208/s12248-012-9350-9.
14. Humayoon R, Razvi N, Anjum F, Ghayas S, Ghazal L, Siddiqui S. Quality Control Testing and Equivalence of Doxycycline Hyclate $(100 \mathrm{mg})$ Capsule Brands under Biowaiver Conditions. J. Pharm Drug Deliv Res. 2014;(32):1-7. doi: $10.4172 / 2325-9604.100124$

15. World Health Organization. General Notes on Biopharmaceutics Classification System (BCS) - based Biowaiver Applications [Internet]. Geneva: WHO; 2014. Disponible en: http://apps.who. int/medicinedocs/documents/s21674en/ s21674en.pdf

16. Guerrero F. Determinación de la Intercambiabilidad Terapéutica de Cápsulas de Fluconazol Comercializadas en el país en relación a su innovador Diflucan ${ }^{\circledR}$ [Tesis] Quito: Facultad de Ciencias Químicas. Universidad Central del Ecuador; 2013. Disponible en http://www.dspace.uce. edu.ec/bitstream/25000/1881/1/TUCE-0008-26.pdf

17. Martin M. The use of fluconazole and itraconazole in the treatment of Candida albicans infections: a review. J Antimicrob Chemother. 1999;44(4):429-37.

18. Dirección General de Medicamentos Insumos y Drogas [Internet]. Lima, DIGEM-
ID; 2018. Registro Sanitario de Productos Farmacéuticos. Disponible en: http:// www.digemid.minsa.gob.pe/ProductosFarmaceuticos/principal/pages/Default. aspx

19. Reglamento que regula la intercambiabilidad de medicamentos. D.S. No 024-2018-SA. Diario Oficial El Peruano (15-09-2018). Disponible en: https:// busquedas.elperuano.pe/download/url/ aprueban-reglamento-que-regula-la-intercambiabilidad-de-medi-decreto-supremo-n-024-2018-sa-1692074-1

20. Organización Panamericana de la Salud. Marco para la Ejecución de los Requisitos de Equivalencia para los Productos Farmacéuticos [Internet]. Washington D.C: OPS; 2011. Disponible en: http:// apps.who.int/medicinedocs/documents/ s22162es/s22162es.pdf

Correspondencia: Luis Moreno Exebio Dirección: Las Higueras 175, Dpto. 201, La Molina. Lima, Perú Teléfono: (511) 996728185

Correo electrónico:lemoreno70@hotmail.com /lmoreno@ins.gob.pe

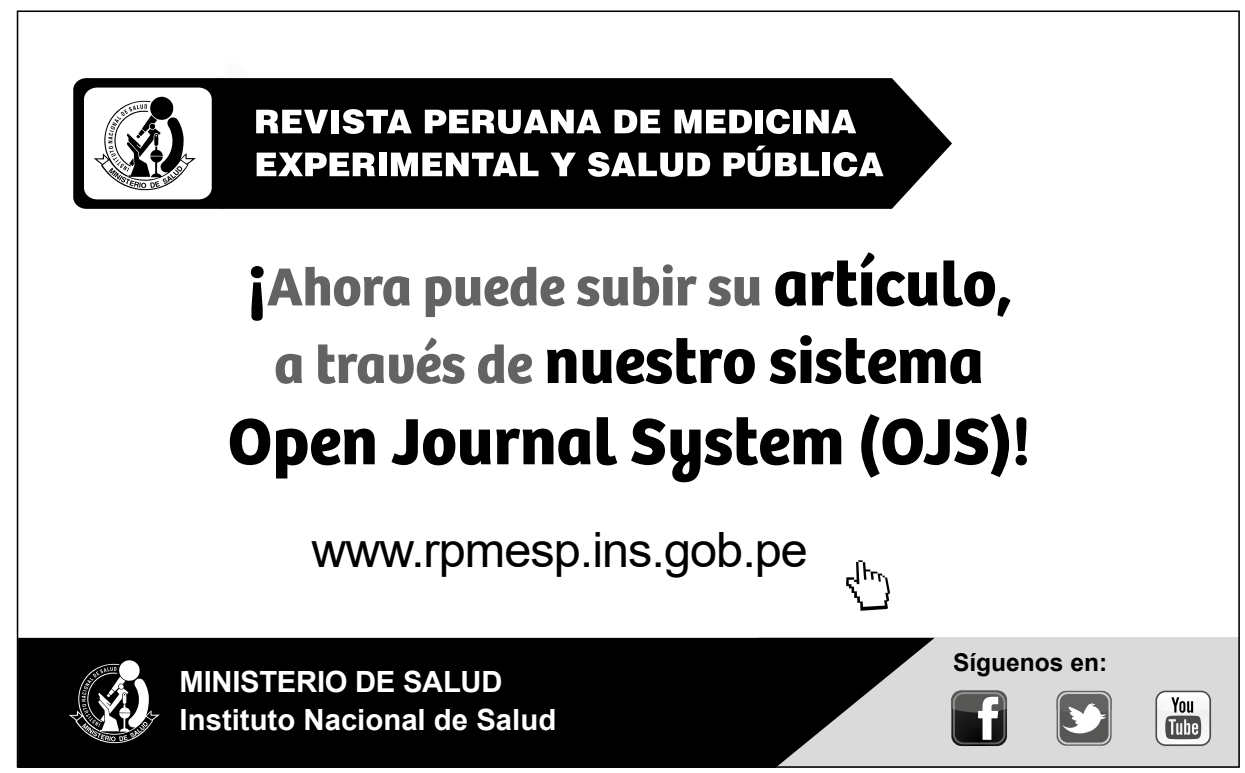

